\title{
Selective Adaptation in Networks of Cortical Neurons
}

\author{
Danny Eytan, ${ }^{1}$ Naama Brenner, ${ }^{2}$ and Shimon Marom ${ }^{1}$ \\ Faculties of ${ }^{1}$ Medicine and ${ }^{2}$ Chemical Engineering, Technion-Israel Institute of Technology, Haifa 31096, Israel
}

\begin{abstract}
A key property of neural systems is their ability to adapt selectively to stimuli with different features. Using multisite electrical recordings from networks of cortical neurons developing ex vivo, we show that neurons adapt selectively to different stimuli invading the network. We focus on selective adaptation to frequent and rare stimuli; networks were stimulated at two sites with two different stimulus frequencies. When both stimuli were presented within the same period, neurons in the network attenuated their responsiveness to the more frequent input, whereas their responsiveness to the rarely delivered stimuli showed a marked average increase. The amplification of the response to rare stimuli required the presence of the other, more frequent stimulation source. By contrast, the decreased response to the frequent stimuli occurred regardless of the presence of the rare stimuli. Analysis of the response of single units suggests that both of these effects are caused by changes in synaptic transmission. By using synaptic blockers, we find that the increased responsiveness to the rarely stimulated site depends specifically on fast GABAergic transmission. Thus, excitatory synaptic depression, the inhibitory sub-network, and their balance play an active role in generating selective gain control. The observation that selective adaptation arises naturally in a network of cortical neurons developing ex vivo indicates that this is an inherent feature of spontaneously organizing cortical networks.
\end{abstract}

Key words: neural networks; excitability; multi-electrode array; neurons; cortex; inactivation; adaptation

\section{Introduction}

Selective adaptation to stimuli with different features is seen in a range of brain-behavior functions and sensory modalities. In particular the frequency of stimuli is one property that can induce selective changes in the sensitivity of the response to those stimuli. This phenomenon has two components: a decline of neuronal responses with time as a result of repetitive presentations of a frequent stimulus and a maintenance of relatively high sensitivity of the response to rarely presented stimuli. It has been named "stimulus-specific adaptation" in the context of visual stimuli and auditory cortex (Ringo, 1996; Ulanovsky et al., 2003), "mismatch negativity" in the context of the event-related potentials field (Tiitinen et al., 1994; Naatanen et al., 2001), and "adaptation-induced plasticity" in the context of orientation tuning in the visual cortex (Dragoi et al., 2000). Neurophysiological correlates to the phenomenon are observed using brain imaging (McCarthy et al., 1997; Clark et al., 2000; Kirino et al., 2000; Opitz et al., 2002), electroencephalography (Picton, 1992; Tiitinen et al., 1994; Naatanen et al., 2001), and intracranial single neuron recordings (Saul and Cynader, 1989; Dragoi et al., 2000, 2001; Sengpiel and Bonhoeffer, 2002; Ulanovsky et al., 2003). Regardless of the exact modality or cortical location, it seems that the various neuronal structures implicated in this phenomenon make use of a mechanism for selective gain control (Abbott et al.,

Received June 17, 2003; revised Aug. 25, 2003; accepted Aug. 29, 2003.

This work was partially funded by research grants from the Israel Science Foundation (S.M.), the National Institute for Psychobiology in Israel (S.M.), the Minerva Foundation (S.M.), and the United States-Israel Binational Foundation (N.B.). We thank Elleonora Lyakhov, Vladimir Lyakhov, Eyal Jacobson, and Amir Minerbi for technical support, Erez Braun, Daniel Dagan, Goded Shahaf, Nachum Ulanovsky, and Noam Ziv for helpful discussions, and Jackie and Yitzhak Schiller for carefully reading this manuscript.

Correspondence should be addressed to Danny Eytan, Department of Physiology, Faculty of Medicine, TechnionIsrael Institute of Technology, P.0. Box 9649, Haifa 31096, Israel. E-mail: dannye@tx.technion.ac.il.

Copyright $\odot 2003$ Society for Neuroscience $\quad 0270-6474 / 03 / 239349-08 \$ 15.00 / 0$
1997), extracting the common invariant input patterns, while remaining sensitive to rare features. It has been suggested that selective adaptation is a network phenomenon rather than a single neuron property (Ringo, 1996; Abbott et al., 1997; Dragoi et al., 2000; Naatanen et al., 2001; Sengpiel and Bonhoeffer, 2002), and attention has focused on synaptic depression in this context (Abbott et al., 1997; Varela et al., 1997; Zucker and Regehr, 2002).

In the last several years, specific cortical structures have been suggested as generators of this phenomenon; however, given the ubiquitous nature of selective adaptation in perception, it is reasonable to expect the existence of general network-level principles that underlie this phenomenon. The present experimental research focuses on the level of neuronal populations in large networks of cortical neurons, seeking an analog of selective adaptation at this level and a characterization of the underlying mechanisms that may support such network behavior. The experimental system is built of random ex vivo networks of cortical neurons (Baughman et al., 1991; Higgins and Banker, 1998). Relevant cortical in vivo features are conserved in these ex vivo networks, including connectivity and distribution of types of cells, as well as synaptic and cellular level plasticity (for review, see Marom and Shahaf, 2002). When developing on an array of electrodes (see Fig. 1A), ex vivo networks can be interrogated by site-specific stimuli with varying temporal and spatial structures. Recent studies (Maeda et al., 1998; Jimbo et al., 1999; Tateno and Jimbo, 1999; Shahaf and Marom, 2001; for review, see Marom and Shahaf, 2002) show that such site-specific stimuli induce pathway-specific (rather then neuron-specific) changes in responsiveness (potentiation or depression), which may last for at least several minutes. Moreover, the observed changes are at the fine structure of the spike trains and correlations between activities that are not necessarily obvious when one looks at global statistics. These findings indicate that ex vivo networks may serve 
for studying interactions between pathways. The present study explores mechanisms of selective adaptation by focusing on interactions between activation pathways evoked by rare and frequent stimulation at two different sites.

\section{Materials and Methods}

Cultured networks. Cortical neurons were obtained from newborn rats within $24 \mathrm{hr}$ after birth, following standard procedures as described previously (Shahaf and Marom, 2001; Marom and Shahaf, 2002). The cortex tissue was enzymatically digested and mechanically dissociated. The neurons were plated directly onto substrate-integrated multi-electrode array (MEA) dishes (Gross, 1979; Stenger and McKenna, 1994) (see below and Fig. $1 A$ ). The cultures were bathed in MEM supplemented with heatinactivated horse serum ( $5 \%)$, glutamine $(0.5 \mathrm{~mm})$, glucose $(20 \mathrm{~mm})$, and gentamycin $(10 \mu \mathrm{g} / \mathrm{ml})$ and maintained in an atmosphere of $37^{\circ} \mathrm{C}, 5 \%$ $\mathrm{CO}_{2}$ and $95 \%$ air in a tissue culture incubator as well as during the recording phases. Experiments were performed during the third week after plating, thus allowing functional and structural maturation of the neurons (Marom and Shahaf, 2002).

Electrophysiology. We used arrays of $60 \mathrm{Ti} / \mathrm{Au} / \mathrm{TiN}$ electrodes, $30 \mu \mathrm{m}$ in diameter and spaced $200 \mu \mathrm{m}$ from each other (MultiChannelSystems, Reutlingen, Germany). The insulation layer (silicone nitride) was pretreated with poly-D-lysine. A commercial 60-channel amplifier (B-MEA1060, MultiChannelSystems) with frequency limits of $1-5000 \mathrm{~Hz}$ and a gain of $1024 \times$ was used. The B-MEA-1060 was connected to MCPPlus variable gain filter amplifiers (Alpha-Omega, Nazareth, Israel) for further amplification. Stimulation through the MEA was performed using a dedicated eight-channel stimulus generator (MultiChannelSystems). Data were digitized using two, parallel 5200a/526 analog-to-digital boards (Microstar Laboratories, Bellevue, WA). Each channel was sampled at a frequency of 24 kilosample/sec and prepared for analysis using the AlphaMap interface (Alpha-Omega). Thresholds ( $8 \times$ rms units, typically in the range of $10-20 \mu \mathrm{V}$ ) were defined separately for each of the recording channels before the beginning of the experiment. All results shown here for dual-site stimulation experiments (see Figs. 2-4, 6-9) are on the basis of data extracted from voltage traces using the principal component spike-sorting technique (Abeles and Goldstein, 1977) (AlphaSort, Alpha-Omega). To minimize confusion between spike sources recorded from the same electrode, only one (best sorted) unit was chosen from each electrode. In single stimulation site experiments (see Fig. 5), data without spike sorting are presented; sample experiments were subjected to spike sorting, and no qualitative differences in the results were detected. The stimulating electrodes were chosen according to their ability to induce a reliable, reverberating electrical activity in response to a biphasic current pulse ( $\leq 60 \mu \mathrm{A} ; \leq 300 \mu$ sec for each phase); these singlestimulus parameters for the two sites in each experiment were identical. The first $3 \mathrm{msec}$ after a stimulus are not included in the analyzed data to exclude stimulus artifacts.

Selective adaptation experiments (two-site stimulation). Seven experiments were conducted in which networks were stimulated from two sites. Presented are results of 182 single units isolated using spike sorting from six experiments. In one experiment we did not observe selective adaptation; this experiment is addressed separately in Results. Each experiment lasted 15 min; 162 stimuli were delivered through the frequently stimulated site, and 18 stimuli were delivered through the rarely stimulated site at $1 / 5$ and $1 / 50 \mathrm{sec}^{-1}$, respectively. Stimulation frequencies for the two sites are dictated on the higher end by the requirement to evoke network inactivation, and on the lower end, not to evoke such response decline (see Fig. 5). In our networks, this range amounts to $>1 / 10 \mathrm{sec}^{-1}$ at the frequently stimulated site and $<1 / 10 \mathrm{sec}^{-1}$ at the rarely stimulated site. Thus, the low frequency $\left(1 / 50 \mathrm{sec}^{-1}\right)$ was selected on the basis of the fact that it does not cause a marked decline of responsiveness in all networks tested. The higher stimulation frequency $\left(1 / 5 \mathrm{sec}^{-1}\right)$ was chosen arbitrarily to be one order of magnitude higher. Stimulation order was set to a 9:1 pattern as shown in Figure $1 A$. In addition to the $1 / 50$ and $1 / 5 \mathrm{sec}^{-1}$ experiments presented here, experiments with other frequencies were conducted $\left(1 / 2-1 / 20 \mathrm{sec}^{-1} ; 1 / 3\right.$ and $\left.1 / 30 \mathrm{sec}^{-1}\right)$ with similar results. Before each experiment, the networks were monitored for at least $15 \mathrm{~min}$ to ensure stability of the activity. In addition, two control experiments in which the networks were stimulated at identical frequencies $(1 / 5$ and $1 / 50 \mathrm{sec}^{-1}$ ) from the two sites were performed (see Fig. 7 A,B).

Single-site stimulation experiments. Twenty-four single site stimulation experiments in 19 different networks were performed to characterize the kinetics of response inactivation (see Fig. 5). Three to six networks were used for each stimulation frequency examined (an average of $\sim 30$ active electrodes for each network). Before each experiment, the networks were monitored for at least $15 \mathrm{~min}$ to ensure stability of the activity.

Synaptic blockade experiments. To explore the mechanism of selective adaptation, eight experiments with specific synaptic blockers were performed. The synaptic blockers used were as follows: (1) 2-amino-5phosphonovalerate (APV), 8-10 $\mu \mathrm{M}$. One experiment was performed using a single stimulation source and the other using the standard selective adaptation protocol; (2) 6,7-dinitroquinozaline-2,3- $(1 \mathrm{H}, 4 \mathrm{H})$ dione) (DNQX), $6-10 \mu \mathrm{M}$, and (3) bicuculline, 0.5-1.5 $\mu \mathrm{M}$. Higher concentrations of synaptic blockers caused either a complete block of propagated activity in the networks (DNQX and APV) or hyperactivity with long-lasting bursts $(>2 \mathrm{sec})$ in response to stimulation and unreliable responsiveness (bicuculline). DNQX was prepared in stock solution in DMSO and diluted in bath application $>2000$-fold.

Data analysis. Post-stimulus time histograms (PSTHs) were calculated using either a $5 \mathrm{msec}$ (see Fig. 2) or $10 \mathrm{msec}$ (see Fig. 3) time bin. Population PSTH (PPSTH) is the sum of all PSTHs obtained for all sorted spike sources in the network ( 36 units in the network shown in Figs. $2 B$ and 3). Responsiveness is defined as the number of spikes detected in a unit within $150 \mathrm{msec}$ after a stimulus. Initial and final responsiveness, for two-site stimulation experiments, are averaged over the first six stimuli delivered and the final six stimuli delivered from each site, respectively. Accordingly, Figure $4 B$ shows only units that responded at least once to one of the first or final six stimuli from each site. In Figures 5 and $6 B$, the change in average network responsiveness for each time point is calculated from the number of spikes evoked by five consecutive stimuli, normalized to the initial average responsiveness.

\section{Results}

Figure 1 shows the experimental setup and paradigm. Each network was stimulated at two different sites (Fig. $1 A$, right): relatively frequent stimuli $\left(1 / 5 \mathrm{sec}^{-1}\right)$ were delivered from site $A$ and relatively rare stimuli $\left(1 / 50 \mathrm{sec}^{-1}\right)$ were delivered from site $\mathrm{B}$. In each experiment, the network was stimulated continuously with 180 stimuli, of which 162 were delivered from the frequently stimulated site (site A) and 18 from the rarely stimulated site (site B). The ratio between the stimulation times of the two sites was set to be 9:1, that is, every nine stimuli from site A were followed by one stimulus from site B. A single stimulus evoked a network reverberating response lasting several tens of milliseconds, detectable by the recording electrodes. There are 60 recording electrodes in this array (Fig. $1 A$, right, gray dots). A voltage trace recorded from one such electrode in response to a stimulus delivered at site $\mathrm{A}$ is shown in the top trace of Figure $1 B$ (concatenated threshold-crossing segments). All recorded signals from all recording electrodes underwent a principal-component spikesorting procedure (Abeles and Goldstein, 1977). A representative extracted spike shape is shown in the bottom trace of Figure $1 B$. This analysis procedure produced activity from several tens of identified spike sources ("units") for each network. As observed by others (Jimbo et al., 2000; Shahaf and Marom, 2001; Marom and Shahaf, 2002), a typical response to stimulation in a random network of cortical neurons includes two distinct phases (Fig. 2). The early phase, which terminates within $\sim 20 \mathrm{msec}$, mostly reflects direct activation of a distinct subset of the neurons in the network whose identity is determined by the stimulating electrodes location. The late "reverberating" phase, which may last hundreds of milliseconds, involves the propagation of signals through multiple and possibly recurrent synaptic pathways in the 
A
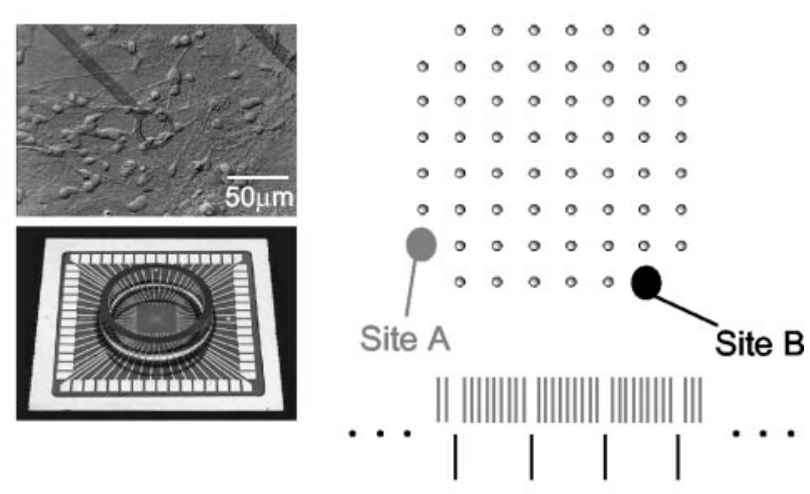

B

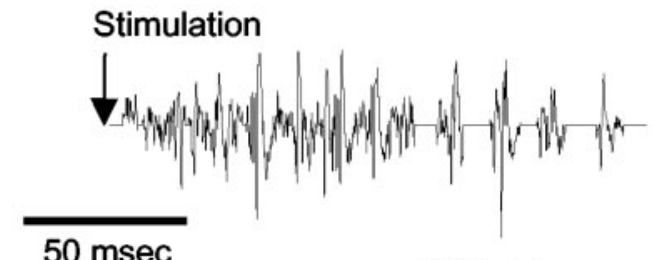

$50 \mathrm{msec}$

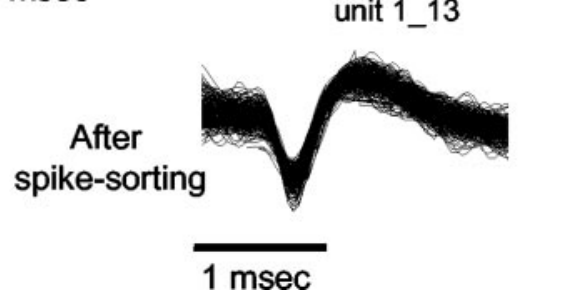

Figure 1. Experimental setup and paradigm. $A$, Experimental setup; a network of cortical neurons developing on a substrate-embedded multi-electrode array. Sites for stimulation are represented schematically by gray $\left(1 / 5 \mathrm{sec}^{-1}\right)$ and black $\left(1 / 50 \mathrm{sec}^{-1}\right)$ circles. B, A typical response to a single stimulus is composed of multiunit (i.e., several neurons) spikes of various shapes; hence, a spike-sorting algorithm is used. Shown are a sample trace recorded from one of the electrodes and the corresponding spike shapes of one unit extracted from this trace over the course of an experiment.

network and is dependent on NMDA receptor transmission. The two phases seem to reflect the biphasic AMPA-NMDA nature of the glutamate synapses in the culture and its dynamics (Jimbo et al., 2000).

\section{The basic observation}

The results obtained from one network are shown in Figure 2. Figure $2 \mathrm{~A}$ shows the average response of an identified unit to a single frequently delivered (gray) and rarely delivered (black) stimulus. The response is presented in the form of a PSTH of instantaneous firing rate as a function of time after stimulus delivery. Figure $2 \mathrm{~A}$ shows a comparison of the PSTHs of a single unit, at the beginning of the experiment (dashed lines) and after 15 min of stimulation (continuous lines). Note that the responses to stimuli coming from the frequently stimulated site markedly decreased, whereas responses to stimuli coming from the rarely stimulated site increased. Figure $2 B$ shows the same phenomenon, represented at the population level (PPSTH), summing firing rates over all the identified spike sources (units) extracted from the network.

The changes in the PSTHs shown in Figure 2 are reversible. This is exemplified in Figure 3. Within 15 min of stimulation in given loci of frequently and rarely stimulating electrodes, the network becomes hypersensitive to the rare and hyposensitive to

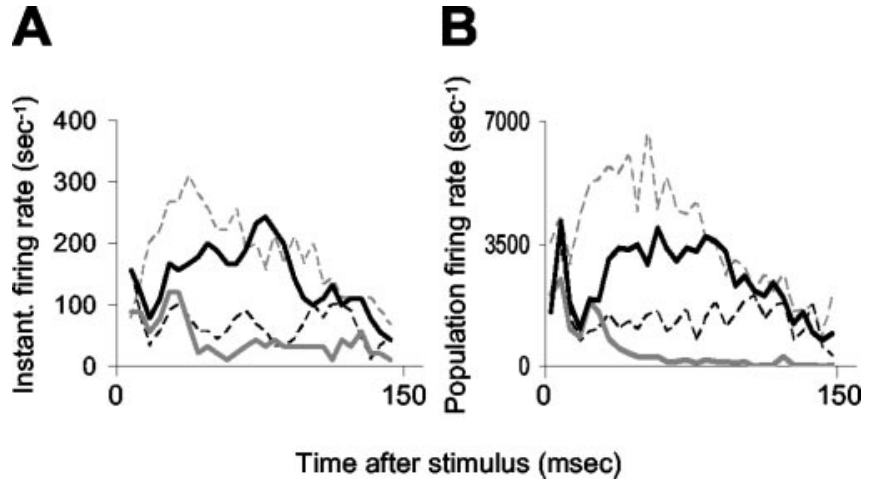

Figure 2. Selective adaptation; single unit and population. A, PSTHs for the unit whose spikes are shown in Figure $1 B$, at the beginning and end of a series of network stimulations from the frequent (gray) and rare (black) sites. Dashed lines depict PSTHs obtained from the first six stimuli of each site; continuous lines depict PSTHs obtained from the last six stimuli of each site. $B$, PPSTH of the same experiment as in $A$ (36 units).

the frequent (left panel). Then, the network is left unstimulated for $15 \mathrm{~min}$, after which the differences in sensitivity to the two sources diminish (middle panel). Thereafter, the frequent and rare stimuli loci are reversed, and stimuli are delivered for an additional $15 \mathrm{~min}$. At the end of this last session, the responses have reversed accordingly (right panel). This reversal of changes in the PSTH was observed in an additional culture and a total of 68 units.

The ability to differentiate between rare and frequent, at the individual neuron level, is presented in Figure 4 (summarizing results from six experiments obtained from four different networks). Figure $4 A$ shows that this property results from two processes. The responsiveness of individual units to frequent stimuli decreases, whereas responsiveness of these same units to the rarely delivered stimuli remains stable or increases. Each point in this graph represents an identified spike source (i.e., one unit). Responsiveness is defined as the number of spikes detected in a unit within $150 \mathrm{msec}$ after a stimulus. Initial and final responsiveness are averaged over the first six stimuli and the final six stimuli delivered from each site, respectively.

Most of the units respond preferentially to the rare stimuli at the end of the experiment, regardless of their initial ratio of responsiveness. We used the rare-to-frequent ratio of responsiveness as a quantitative measure of this effect. As can be seen in Figure $4 B$, at the beginning of the experiment ( $x$-axis) many of the units responded similarly to both stimulation sources (ratio $\sim 1$ ). At the end of the experiment ( $y$-axis), almost all points were located above the value of 1 . Thus, the result shown in Figure 2 is observed in identified spike sources from several experiments (182 units, six experiments).

\section{Decline of response to frequent stimulation}

The decrease in responsiveness of the network to the frequent stimulation occurs regardless of the presence of the rare stimulation. Figure $5 A$ shows the slow changes in network response to a continuous train of pulses from one source, at different frequencies. Each point represents a normalized average response to five consecutive stimuli, calculated over several networks (for details, see Fig. 5 legend). As the stimulation frequency increases, the network shows a stronger decrease on a time scale of a few minutes. In some networks, several cycles of response inactivation and partial recovery ("escapes") are observed, as reflected in Figure 5 for frequencies above $0.1 \mathrm{sec}^{-1}$. Figure $5, B$ and $C$, 
shows traces from individual networks of changes in response to stimulation at $1 / 3$ and $1 / 5 \sec ^{-1}$, respectively. The mechanism underlying these partial escapes from inactivation awaits further exploration.

In a network configuration, a decline of response to frequent stimulation can arise from neuronal excitability changes, synaptic changes, or a combination of both. In our experiments, the changes in responsiveness of single units are not an intrinsic property of the unit but depend on the stimulus identity (Fig. $4 A$ ). We have not detected single identified units that significantly decrease their responsiveness to rare stimuli despite the marked attenuation in the response of these same units to the frequent stimulation. This suggests that inactivation in our experiments is mostly a result of short-term synaptic depression (Varela et al., 1997; for review, see Zucker and Regehr, 2002).

To pinpoint the excitatory synaptic resource undergoing depression, we performed several experiments with application of specific NMDA and AMPA receptor blockers (APV and DNQX, respectively). Although both caused a dose-dependent decrease in observed responsiveness to stimulations, selective blockade of NMDA-mediated transmission had a greater effect on the later phases of the response, as seen by others (Jimbo et al., 2000). The average firing rate of the network within the early phase (3-15 msec after the stimulation) was $1292 \pm 282$ spikes per second (mean $\pm \mathrm{SD}$ ) in control conditions and $1368 \pm 607$ spikes per second after the addition of APV. In contrast, within the late phase of the response (50-150 msec) we measured $1885 \pm 1064$ spikes per second before and $54 \pm 216$ spikes per second after exposure to APV $(8-10 \mu \mathrm{M})$. Data are obtained from two networks, 69 units, and 20 consecutive presentations of the stimulus under each condition.

Note that the reverberating response to stimulation is dependent on both excitatory receptor types with complex interactions between them; therefore, it is not possible to isolate the individual contribution of NMDA and AMPA receptors to the transmission depression in frequently activated pathways.

\section{Enhanced response to rare stimulation in the presence of a frequent source}

Increased sensitivity to rarely delivered stimuli is observed only in the presence of frequent stimulation. This is evident from the data presented in Figure 6. Figure $6 \mathrm{~A}$ shows the distribution of the change in responsiveness to rarely delivered stimuli in the presence (black) and absence (gray) of frequently delivered stimuli. Apparently, the presence of the frequent stimulation markedly skews the distribution toward increased responsiveness $(2.35 \pm 3$ as compared with $-0.44 \pm 1.8)$; the two distributions are significantly different $(p<0.001$; Wilcoxon Rank Sum test; $n=442$; six experiments in the presence of frequent stimulation and five without). In Figure $6 B$, the kinetics of change in responsiveness to rarely delivered stimuli in the presence (black circles) and absence (gray squares) of frequently delivered stimuli are shown. Note that the increase in sensitivity takes several minutes to develop and occurs only in the presence of the frequent stimuli. Figure $7, A$ and $B$, shows the results of a control experiment in which the two sites are stimulated at the same frequency: 1/50 or $1 / 5 \mathrm{sec}^{-1}$. Under such conditions, the response of units to fre-

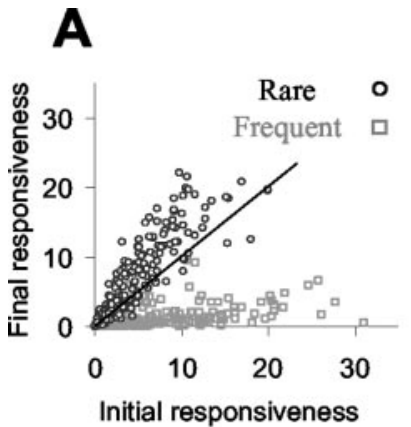

B

Time after stimulus (msec)

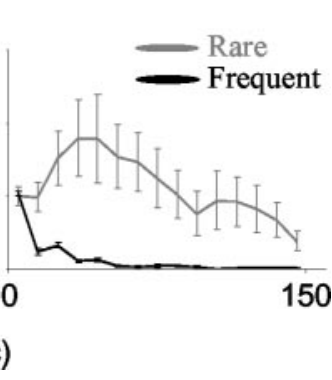

150

Figure 4. Selective adaptation for each unit is composed of two components: decreased response to the frequently stimulated site and an average increased response to the rarely stimulated site. In $A$, final versus initial responsiveness to the rarely (black circles) and frequently (gray squares) stimulated sites is shown for each unit (182 units). Responsiveness is defined as the mean number of spikes detected in a unit within 150 msec after a stimulus. Initial and final responsiveness are averaged over the first and last six stimuli delivered from each site, respectively. The equivalence line is plotted in black. Both average increased responsiveness to the rarely stimulated site and average decreased responsiveness to the frequently stimulated site are statistically significant ( $p<0.001$; one-sided Student's $t$ tests). In $B$, the ratio of responsiveness to stimuli from rare/frequent stimulated sites is plotted for each unit (148 units; only those units that responded at least once to 1 of the 6 stimuli from each site) at the beginning ( $x$-axis) and end ( $y$-axis) of the experiment. Although the average initial rare/frequent ratio was 0.91 , the final ratio was 10.85 , representing a significant increase ( $p<0.001$; Wilcoxon signed rank test, calculated using the logarithm of the ratio distributions).

quently delivered stimuli from both sites decreases significantly (Fig. 7A). In contrast, the responsiveness of units to rare $(1 / 50$ $\mathrm{sec}^{-1}$ ) stimuli from both sites (Fig. $7 B$ ) remains relatively stable or even decreases but certainly does not increase, as seen in the above experiments in which rare stimuli were delivered in the presence of frequent ones (compare with Figs. 4A, 6A).

Several pieces of evidence indicate that response inactivation to the frequent stimuli plays a role in the enhancement of sensitivity to the rare. First, the time course of inactivation at a stimulation frequency of $1 / 5 \mathrm{sec}^{-1}$ is similar to the time course of increased sensitivity to rarely delivered stimuli, as seen in Figures 5 and $6 \mathrm{~B}$. It takes the network a few minutes to develop the differentiation between the rare and the frequent stimuli, which is in the same order of time it takes the network to enter an inactivated phase. Second, in those experiments in which enhanced sensitivity to the rare was observed (six of seven; see Materials and Methods), we find a significant negative correlation between the change in responsiveness to the frequently stimu- 


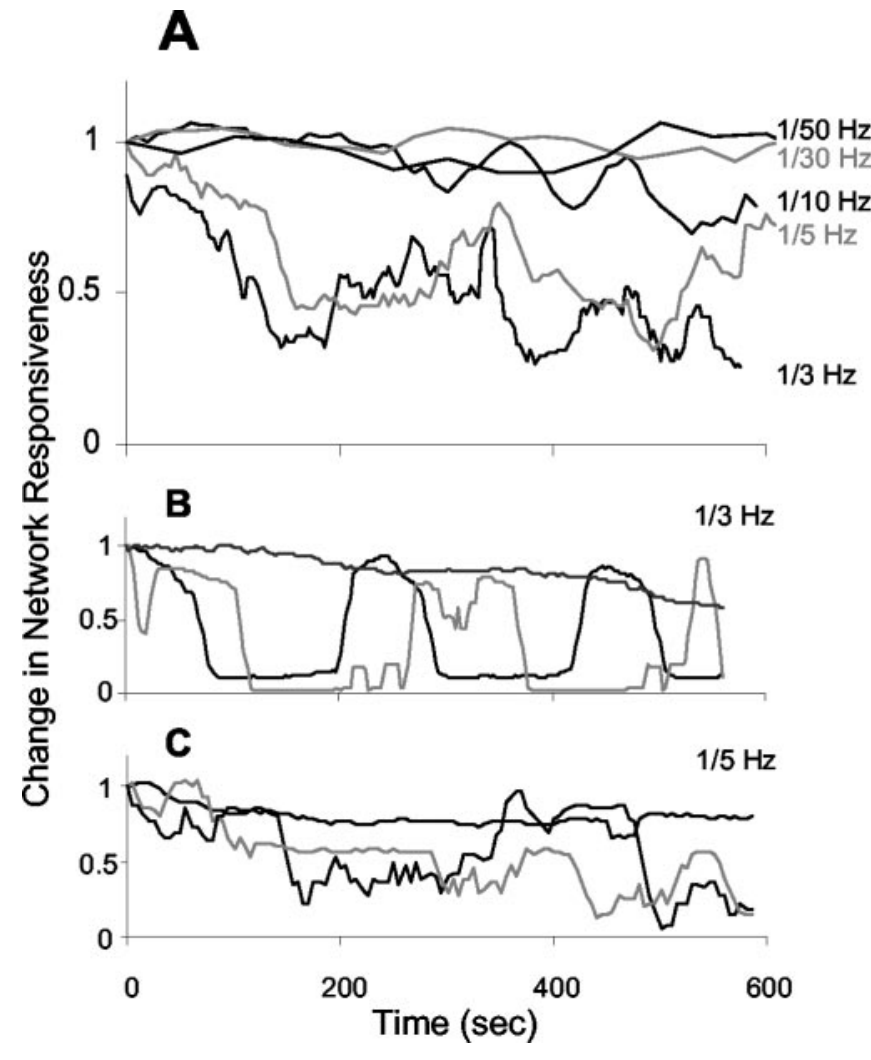

Figure 5. $A$, Changes in network response to continuous stimulation at different frequencies. Each point is calculated from the number of spikes evoked by the entire network in response to five consecutive stimuli normalized to unity at the beginning of the experiment. [Twenty-four single-site stimulation experiments in 19 different networks were performed to characterize the kinetics of inactivation response; each trace represents an average of $n$ experiments $\left(1 / 50 \mathrm{sec}^{-1}, n=5 ; 1 / 30 \mathrm{sec}^{-1}, n=6 ; 1 / 10 \mathrm{sec}^{-1}, n=4 ; 1 / 5 \mathrm{sec}^{-1}, n=4 ; 1 / 3\right.$ $\left.\sec ^{-1}, n=5\right)$; an average of $\sim 30$ active electrodes for each network.] $B$, C, Sample individual traces obtained from six networks showing the changes in response to continuous stimulation at $1 / 3 \mathrm{sec}^{-1}$ ( $B, 3$ different networks) and $1 / 5 \mathrm{sec}^{-1}$ ( $C, 3$ different networks). Same calculation method as in $A$ was used.

lated site and the amplification of the response of the same unit to the rarely stimulated site (Fig. $7 C)(r=-0.694 ; p<0.001$; calculated from all experiments and from all units; $n=182$ ). In other words, the greater the decrease in the response to the frequent stimulation, the larger the amplification to the rare stimuli. Indeed, in the only case in which the network did not inactivate in response to the frequently delivered stimuli, we were also unable to detect an increase in sensitivity to the rarely delivered stimuli (one of seven experiments; see Materials and Methods).

As shown above, the enhanced response to the rarely stimulated site is dependent on the presence of frequent stimulation. To explore the possibility that GABAergic synaptic depression plays a role in the enhancement, we performed selective adaptation experiments in the presence of partial blockade of the fast GABAergic transmission by bicuculline. Figure $8 \mathrm{~A}$ depicts the final versus initial responsiveness to the rarely stimulated site in selective adaptation experiments performed in either the absence of bicuculline (gray circles; same units shown in Fig. $4 B ; n=182$; six experiments) or the presence of $0.5-1.5 \mu \mathrm{M}$ bicuculline (black squares; $n=156$; five experiments). It is evident that addition of bicuculline markedly reduces the enhancement of the response to the rarely stimulated site. To quantify this effect we performed additional analysis, as can be seen in Figure 8 . This panel shows the percentage of units that enhanced their response to the rarely

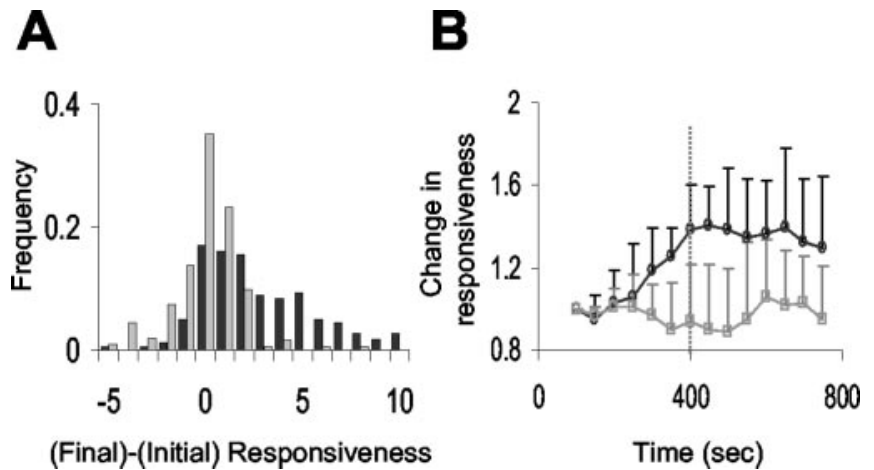

Figure 6. Increased sensitivity to the rare is dependent on frequent stimulation at another site. $A$, Distribution of change in responsiveness (difference between final and initial responsiveness) in the presence (black; $2.35 \pm 3$ ) and absence (gray; $-0.44 \pm 1.8$ ) of another, frequently stimulated site ( $n=442 ; 6$ experiments in the presence of frequent stimulation and 5 without). $B$, Same experiments as in $A$, showing the kinetics of increased sensitivity to the rare in the presence of frequent stimuli (black circles), compared with the relative stability of responsiveness to rare stimuli in the absence of frequent stimuli (gray squares). The change in average network responsiveness for each time point is calculated from the number of spikes evoked by five consecutive stimuli, normalized to the initial average responsiveness of each entire network. Error bars represent SD. When considering all points from 400 sec onward (dotted line), the change in responsiveness in the presence of another, frequent stimulation is significantly higher than the change in responsiveness in the absence of such frequent stimulation ( $p<$ $0.001 ; t$ test).

stimulated site after 15 min of stimulation. Although in the selective adaptation experiments described above $72 \%$ of the units increased their responsiveness (six experiments; 182 units; see also Fig. $6 \mathrm{~A}$ ), in the presence of $0.5 \mu \mathrm{m}$ bicuculline we observed an increase in 55\% of the units (two experiments; 65 units; $p<$ 0.01 compared with the fraction observed without bicuculline); in the presence of $1.5 \mu \mathrm{M}$ bicuculline such an increase was observed in only $36 \%$ (three experiments; 91 units; $p<0.001$ compared with the fraction observed without bicuculline). The fraction of units that increased their responsiveness in the presence of $1.5 \mu \mathrm{M}$ bicuculline is strikingly similar to the fraction observed under stimulation from only one site (at the "rare" frequency of $1 / 50 \mathrm{sec}^{-1} ; p>0.5$ compared with the fraction observed with 1.5 $\mu \mathrm{M}$ bicuculline). Evidently, bicuculline reduces the percentage of units that enhance their responses to rare stimuli to levels seen in stimulation from one source at $1 / 50 \mathrm{sec}^{-1}$ (representing the stable alternative). Figure $8 C$ shows the changes in responses of these same units to the frequent stimulation source. In the presence of both 0.5 and $1.5 \mu \mathrm{M}$ bicuculline, the percentage of units decreasing their responses is significantly higher than that expected under the relatively stable conditions of a slow stimulation source (53\%; white bar). Note that the decreased responsiveness observed is smaller compared with bicuculline-free conditions.

\section{Discussion}

We have shown that the ability to adapt selectively to different stimuli arises naturally in a random network of cortical neurons developing ex vivo with no predefined specialized structure. When stimulated at two sites, the neurons in the network amplified their response to the rarely delivered stimuli and attenuated their response to the more frequent input.

\section{Enhanced response to rare stimulation in the presence of a frequent source}

The observation that the enhanced response to the rarely stimulated site is dependent on the presence of frequent stimulation, taken together with reported properties of the cortical inhibitory 


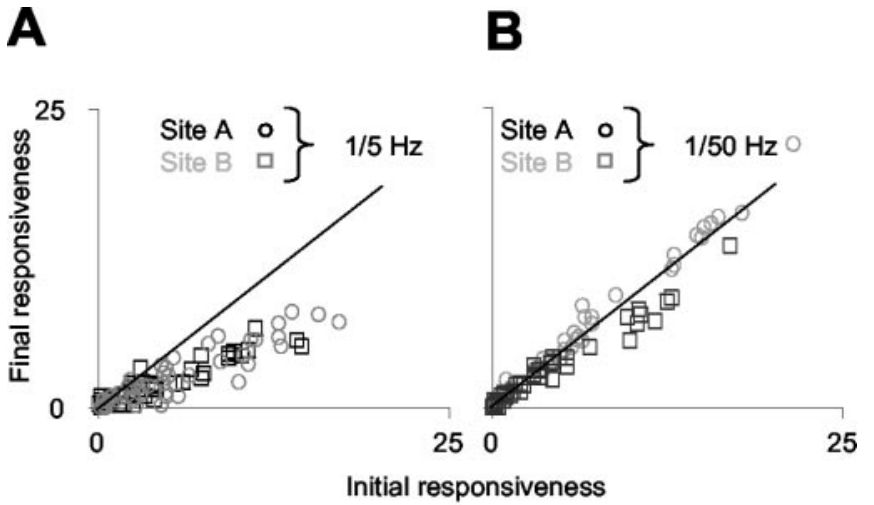

C

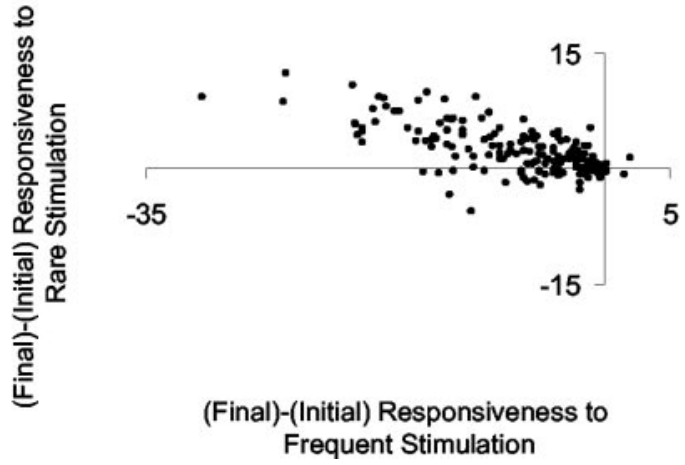

Figure 7. $A, B$, Responsiveness to two equally frequent stimuli; each identified spike source (unit) is depicted by a single point. Average responsiveness of each unit to the first and last six stimuli from site $A$ is presented in black and from site B in gray. Stimulation time was 15 min, similar to that of the selective adaptation experiments. $A$, Sites $A$ and $B$ are stimulated at a rate of $1 / 5 \mathrm{sec}^{-1}$ ( 1 culture; 49 units). The null hypothesis in this case is that there is no change in responsiveness. The results show an average decrease in responsiveness: -2.2 and -2.7 spikes per unit for stimulation from sites $A$ and $B$, respectively; both are statistically significant $\left(p<0.001\right.$; paired $t$ test). B, Both sites are stimulated at a rate of $1 / 50 \mathrm{sec}^{-1}$ ( 1 culture; 46 units). The null hypothesis here is that there is an increase in responsiveness (as is the case of rare in the presence of frequent stimulation); the results show a slight decrease in average responsiveness for both sites $(-0.07 ;-0.95$ spikes per unit; $p=0.24$ and $p<0.01$, respectively). C, Relations between declined response to frequent stimulation and increased response to rare stimulation. The change in responsiveness to the rarely stimulated site [(Final) (Initial) Responsiveness to Frequent Stimulation], for each unit, is shown as a function of the change for the frequently stimulated site $(n=182$; calculated from all selective adaptation experiments).

sub-network, led us to hypothesize a possible role for the inhibitory cells in response enhancement. Because these cells form a highly interconnected (by chemical and electric synapses) network (Beierlein et. al, 2000; for review, see Galarreta and Hestrin, 2001; Voigt et al., 2001), it is reasonable to assume that in contrast to the excitatory activation pathways, which are relatively sensitive to the site of stimulation, the inhibitory activation pathways are not. This implies that the inhibitory sub-network is a common resource. Taken together with activity-dependent shortterm partial depression of fast GABAergic synapses (Galarreta and Hestrin, 1998; Varela et al., 1999), a possible mechanism underlying the enhancement of the response to the rarely stimulated site is suggested: the activation of the frequently stimulated site results in depression of both the excitatory and inhibitory synapses in the pathway stemming from this site. Because excitatory synapses tend to undergo deeper depression than inhibitory ones, the balance between excitation and inhibition for the frequently activated pathway is disrupted, resulting in a marked reduced responsiveness; however, because most of the synapses
A

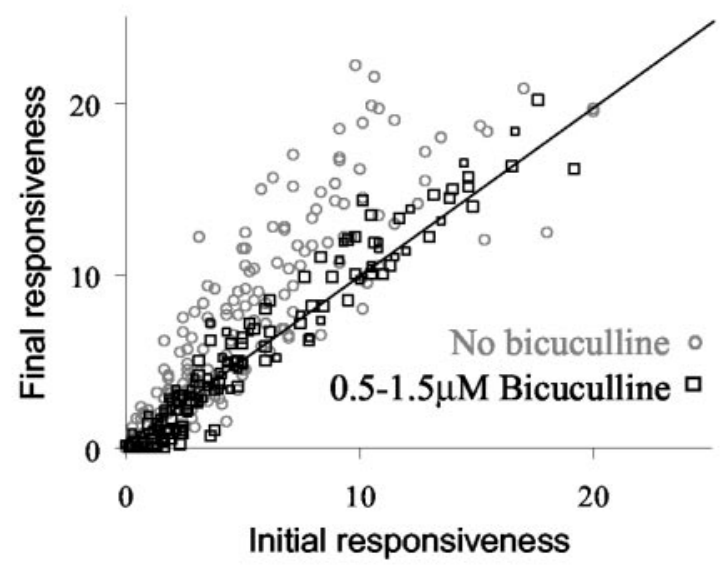

B
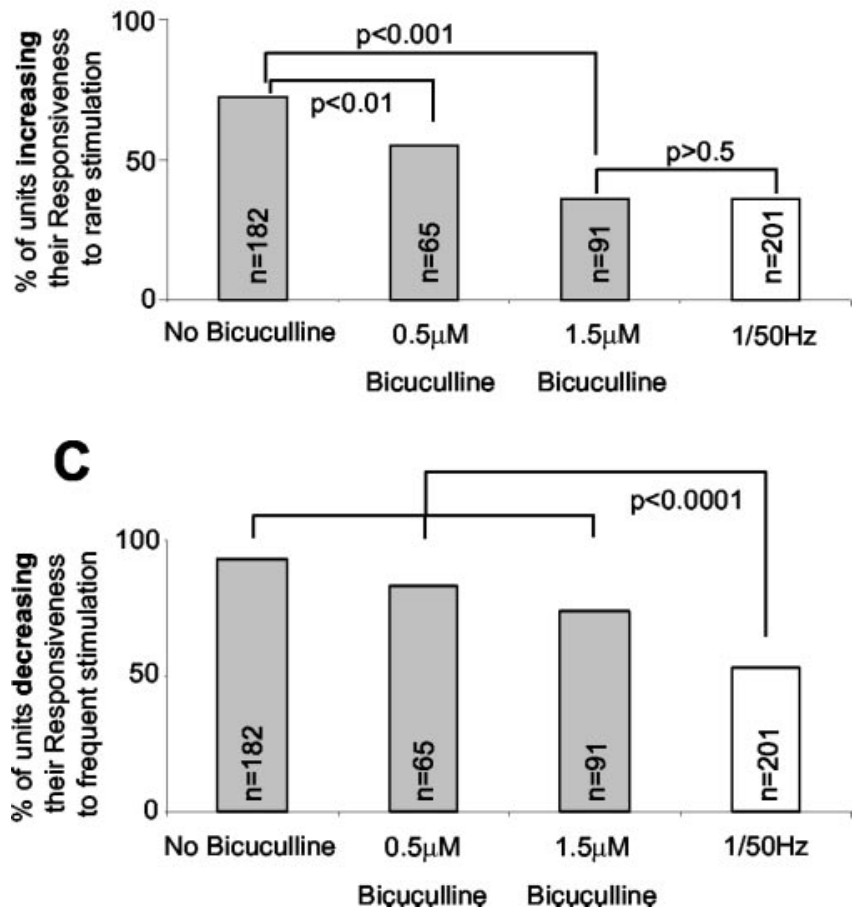

Figure 8. Blocking fast GABAergic synaptic transmission abolishes enhanced sensitivity to rare stimuli seen in selective adaptation. $A$, Final versus initial responsiveness to the rarely stimulated site, for each unit, in selective adaptation experiments performed either in the absence of bicuculline (gray circles; same units shown in Fig. $4 A ; n=182 ; 6$ experiments) or in the presence of $0.5-1.5 \mu \mathrm{m}$ bicuculline (black squares; $n=156 ; 5$ experiments). Initial and final responsiveness are averaged over the first and last six delivered stimuli, respectively. Equivalence line is plotted in black. B, Shown are percentages of units that have enhanced their response to the rarely stimulated site at the end of $15 \mathrm{~min}$ of stimulation, as a function of bicuculline concentration (gray bars). For comparison the same quantity is shown for stimulation at a low frequency $\left(1 / 50 \mathrm{sec}^{-1}\right)$ from one site only (white bar): in the absence of bicuculline, $72 \%$ ( 6 experiments; 182 units; see also Fig. $7 A$ ); $0.5 \mu$ m bicuculline, $55 \%$ (2 experiments; 65 units); in the presence of $1.5 \mu \mathrm{m}$ bicuculline, $36 \%$ (3 experiments; 91 units). $p$ values are calculated using the binomial distribution. C, Shown are percentages of units that have decreased their response to the frequently stimulated site at the end of $15 \mathrm{~min}$ of stimulation, as a function of bicuculline concentration (gray bars). For comparison the same quantity is shown for stimulation at a low frequency $\left(1 / 50 \mathrm{sec}^{-1}\right)$ from one site only (white bar), representing the null case of a relatively stable response: in the absence of bicuculline, 92\% (6 experiments, 182 units; see also Fig. 7A); $0.5 \mu \mathrm{m}$ bicuculline, 83\% (2 experiments; 65 units); in the presence of $1.5 \mu \mathrm{m}$ bicuculline, $74 \%$ (3 experiments; 91 units); stimulation at a low frequency $\left(1 / 50 \mathrm{sec}^{-1}\right), 53 \%$ (5 experiments; 201 units). $p$ values are calculated using the binomial distribution. 
comprising the excitatory pathway from the rarely stimulated site are not depressed whereas the overall inhibitory resources are, the response to the rare stimulation source is increased on average. The results shown above support this interpretation: addition of bicuculline, a $\mathrm{GABA}_{\mathrm{A}}$ antagonist, leads to a reduction in the fraction of units that increase their responsiveness to the rare stimulation source. Moreover, such a blockade of fast inhibitory synaptic transmission is enough to reduce the enhancement down to the level observed with rare stimulation without the presence of a frequent source. Such an interpretation is also compatible with the dependency of the increased responsiveness to the rarely stimulated site on another, more frequent site and with the negative correlation between the change in responsiveness to the frequently stimulated site and the amplification of the response of the same unit to the rarely stimulated site.

That units in these networks can increase their responsiveness to focal stimuli is not a new observation (Maeda et al., 1998; Jimbo et al., 1999; for review, see Marom and Shahaf, 2002). In all reported cases, however, such increased responsiveness was achieved by transient tetanization (at the range of tens of cycles per second). In the present study we show that selective potentiation of responses to rare stimuli can be achieved, provided the network is "primed" by higher (but not tetanizing) frequencies from another site.

\section{Decline of response to frequent stimulation}

In our experiments, a decrease in response of the entire network was observed when the network was stimulated at frequencies higher than $\sim 0.1 \mathrm{sec}^{-1}$ (Fig. 5). It is important to note that the stimulation frequency is not synonymous with the neuronal firing frequency. Each stimulus evokes a reverberating network response in which some of the neurons may fire at instantaneous rates as high as several hundreds of spikes per seconds. As shown in Figure 5, at stimulation frequencies lower than $\sim 0.1 \mathrm{sec}^{-1}$, there is enough time for the effects of responses to relax, and no accumulation of inactivation occurs. The stimulation frequencies shown here to induce declined network response are in accord with reports by others, using this preparation and in the context of stimulation from one site. For example, Maeda et al. (1998) used a stimulation frequency of $1 / 15-1 / 30 \mathrm{sec}^{-1}$ to allow recovery from response decline over time; Jimbo et al. (1993) reported that the networks cannot follow periodic stimulation separated by $<3 \mathrm{sec}$. Shahaf and Marom (2001) reported that at stimulation frequencies $>1 \mathrm{sec}^{-1}$ the networks usually inactivate after a few pulses. Although decreased responsiveness is seen also at the level of the single isolated neurons in this preparation (Tal et al., 2001), cellular excitability does not seem to be a key player in this network phenomenon. The fact that we have not detected single identified units that significantly decrease in responsiveness to rare stimuli (Fig. 4) despite the response of those same units to the frequent stimulation being markedly attenuated suggests that inactivation in our experiments is mostly a result of short-term synaptic depression rather than cellular-level excitability reduction. A similar line of reasoning is suggested by Ringo (1996) and analyzed theoretically by Abbott et al. (1997).

The above interpretation of the data is supported further by the observation that decreased responsiveness to the frequently stimulated site occurs also in the presence of bicuculline, but to a lesser extent. Under normal conditions the balance between excitation and inhibition for the frequently activated pathway is disrupted in favor of inhibition; hence, blockade of fast inhibition partially decreases the extent of response decline.

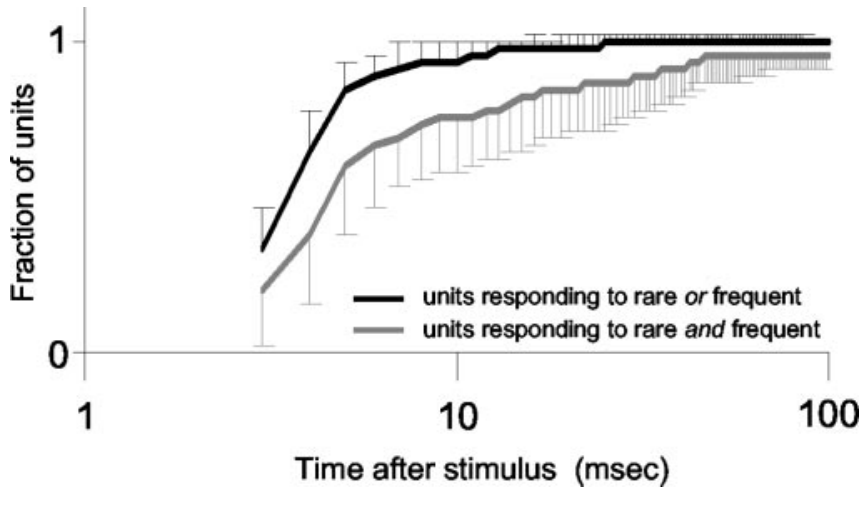

Figure 9. Differential recruitment of neurons by two different stimulation sites. Time (milliseconds) is shown relative to stimulation. The average of four different networks is shown (213 sorted units were examined for their participation in 6 consecutive stimuli from each site). Black line depicts the cumulative fraction of units that responded to either one of the stimulation sources, providing indication for the total rate of neuronal recruitment by a stimulus. The gray line depicts the cumulative fraction of units that responded to both stimulation sites at least once, providing an indication of the potential overlap between the activation pathways evoked by the two stimulation sources. Error bars represent SD (one sided, for clarity).

\section{Activation paths}

Placing the relevant kinetics underlying selective adaptation at the level of the synapses entails that it is not the identity of the units participating in the response that is important but the path defined by the activated synapses. Because the stimulation frequency from the two sources in our experiments is different, these two sources can induce separate effects on the activity of the population (Jimbo et al., 1999). Exemplifying this is Figure 9, which shows the cumulative fraction of units responding to either the rare or frequent stimulus as a function of time after stimulation delivery (black line) as well as the cumulative fraction of units responding to frequent and rare (gray line). Beyond $\sim 25$ msec after a stimulus, the two different stimulation sources activate the same neurons. Thus the mechanism implying a role for GABAergic synapses in response enhancement can be described in terms of interaction between such activation paths. From Figure 9 we learn that each path may be roughly divided into two segments; an early segment, which is unique to each of the paths, and a later segment, in which some overlap between the two paths exists. The fact that the frequently stimulated path is inactivated suggests that the efficacy of signal propagation in one of its segments, or both, decreases. On the other hand, the fact that the rarely stimulated path increases its responsiveness suggests that the efficacy of signal propagation in one of its segments, or both, increases. This suggests that the propagation of the rarely delivered signal is enhanced in the late common segment (but not in the early unique segment) by the frequent stimulation. Indeed, Figures 2, $A$ and $B$, and 3 show that the increased sensitivity to the rare is mostly confined to the later phase of the response.

In summary, we have shown that for simple input patterns the phenomenon of selective adaptation arises naturally in a spontaneously organizing network of heterogeneous neurons. When stimulated at two sites, the network amplifies its responsiveness to the rarely delivered stimuli and attenuates its responsiveness to the more frequent input. The differential change in responsiveness to the two stimulation sources occurs gradually over several minutes (Fig. $6 B$ ) and is restricted to the later phase of the response (Figs. 2A, $B, 3$ ). These features are reminiscent of the results observed in human experiments (for review, see Naatanen et al., 2001) as well as in experiments in which intracranial neuronal 
activity was recorded (Saul and Cynader, 1989; Ringo, 1996; Dragoi et al., 2000, 2001; Sengpiel and Bonhoeffer, 2002; Ulanovsky et al., 2003) (see Introduction). Dissection of the synaptic loci underlying this phenomenon points to depression of the excitatory synaptic transmission at the base of the decreased response to frequent stimulation. This is in accord with the interpretation by Abbott et al. (1997). Depression of the fast GABAergic transmission seems to underlie the enhanced response to the rare stimulation source. Thus, excitatory synaptic depression, the inhibitory sub-network, and their balance play an active role in the generation of this phenomenon. Taken together, these results indicate that selective adaptation is an inherent feature of selforganizing cortical networks, stemming from the properties of cells and their synaptic connections.

\section{References}

Abbott LF, Varela JA, Sen K, Nelson SB (1997) Synaptic depression and cortical gain control. Science 275:220-224.

Abeles M, Goldstein MH (1977) Multispike train analysis. Proc IEEE 65:762-773.

Baughman RW, Huettner JE, Jones KA, Khan AA (1991) Cell culture of neocortical and basal forebrain from postnatal rats. In: Culturing nerve cells (Banker G, Goslin K, eds), pp 227-250. Cambridge, MA: MIT.

Beierlein M, Gibson JR, Connors BW (2000) A network of electrically coupled interneurons drives synchronized inhibition in neocortex. Nat Neurosci 3:904-910.

Clark VP, Fannon S, Lai S, Benson R, Bauer L (2000) Responses to rare visual target and distractor stimuli using event-related fMRI. J Neurophysiol 83:3133-3139.

Dragoi V, Sharma J, Sur M (2000) Adaptation-induced plasticity of orientation tuning in adult visual cortex. Neuron 28:287-298.

Dragoi V, Rivadulla C, Sur M (2001) Foci of orientation plasticity in visual cortex. Nature 411:80-86.

Galarreta M, Hestrin S (1998) Frequency-dependent synaptic depression and the balance of excitation and inhibition in the neocortex. Nat Neurosci 1:587-594.

Galarreta M, Hestrin S (2001) Electrical synapses between GABA-releasing interneurons. Nat Rev Neurosci 2:425-433.

Gross GW (1979) Simultaneous single unit recording in vitro with a photoetched laser deinsulated gold multimicroelectrode surface. IEEE Trans Biomed Eng 26:273-279.

Higgins D, Banker G (1998) Primary dissociated cell cultures. In: Culturing nerve cells (Banker G, Goslin K, eds), pp 37-78. Cambridge MA: MIT.

Jimbo Y, Robinson HP, Kawana A (1993) Simultaneous measurement of intracellular calcium and electrical activity from patterned neural networks in culture. IEEE Trans Biomed Eng 40:804-810.

Jimbo Y, Tateno T, Robinson HP (1999) Simultaneous induction of pathway-specific potentiation and depression in networks of cortical neurons. Biophys J 76:670-678.

Jimbo Y, Kawana A, Parodi P, Torre V (2000) The dynamics of a neuronal culture of dissociated cortical neurons of neonatal rats. Biol Cybern $83: 1-20$.
Kirino E, Belger A, Goldman-Rakic P, McCarthy G (2000) Prefrontal activation evoked by infrequent target and novel stimuli in a visual target detection task: an event-related functional magnetic resonance imaging study. J Neurosci 20:6612-6618.

Maeda E, Kuroda Y, Robinson HP, Kawana A (1998) Modification of parallel activity elicited by propagating bursts in developing networks of rat cortical neurones. Eur J Neurosci 10:488-496.

Marom S, Shahaf G (2002) Development, learning and memory in large random networks of cortical neurons: lessons beyond anatomy. Q Rev Biophys 35:63-87.

McCarthy G, Luby M, Gore J, Goldman-Rakic P (1997) Infrequent events transiently activate human prefrontal and parietal cortex as measured by functional MRI. J Neurophysiol 77:1630-1634.

Naatanen R, Tervaniemi M, Sussman E, Paavilainen P, Winkler I (2001) "Primitive intelligence" in the auditory cortex. Trends Neurosci 24:283-288.

Opitz B, Rinne T, Mecklinger A, von Cramon DY, Schroger E (2002) Differential contribution of frontal and temporal cortices to auditory change detection: fMRI and ERP results. NeuroImage 15:167-174.

Picton TW (1992) The P300 wave of the human event-related potential. J Clin Neurophysiol 9:456-479.

Ringo JL (1996) Stimulus specific adaptation in inferior temporal and medial temporal cortex of the monkey. Behav Brain Res 76:191-197.

Saul AB, Cynader MS (1989) Adaptation in single units in visual cortex: the tuning of aftereffects in the spatial domain. Vis Neurosci 2:593-607.

Sengpiel F, Bonhoeffer T (2002) Orientation specificity of contrast adaptation in visual cortical pinwheel centres and iso-orientation domains. Eur J Neurosci 15:876-886.

Shahaf G, Marom S (2001) Learning in networks of cortical neurons. J Neurosci 21:8782-8788.

Stenger DA, McKenna TM (1994) Enabling technologies for cultured neural networks. London: Academic.

Tal D, Jacobson E, Lyakhov V, Marom S (2001) Frequency tuning of inputoutput relation in a rat cortical neuron in vitro. Neurosci Lett 300:21-24.

Tateno T, Jimbo Y (1999) Activity-dependent enhancement in the reliability of correlated spike timings in cultured cortical neurons. Biol Cybern 80:40-55.

Tiitinen H, May P, Reinikainen K, Naatanen R (1994) Attentive novelty detection in humans is governed by pre-attentive sensory memory. Nature 372:90-92.

Ulanovsky N, Las L, Nelken I (2003) Processing of low-probability sounds by cortical neurons. Nat Neurosci 6:391-398.

Varela JA, Sen K, Gibson J, Fost J, Abbott LF, Nelson SB (1997) A quantitative description of short-term plasticity at excitatory synapses in layer $2 / 3$ of rat primary visual cortex. J Neurosci 17:7926-7940.

Varela JA, Song S, Turrigiano GG, Nelson SB (1999) Differential depression at excitatory and inhibitory synapses in visual cortex. J Neurosci 19:4293-4304.

Voigt T, Opitz T, de Lima AD (2001) Synchronous oscillatory activity in immature cortical network is driven by GABAergic preplate neurons. J Neurosci 21:8895-8905.

Zucker RS, Regehr WG (2002) Short-term synaptic plasticity. Annu Rev Physiol 64:355-405. 